\title{
Video-assisted thoracoscopic surgery right upper lobectomy in a situs inversus totalis patient
}

\author{
Minhua Ye, Jianfei Shen, Min Kong, Jiang Lin, Xiaodong Li, Baofu Chen \\ Department of Cardiothoracic Surgery, Taizhou Hospital of Zhejiang Province, Wenzhou Medical University, Linhai 318000, China \\ Correspondence to: Baofu Chen. Department of Cardiothoracic Surgery, Taizhou Hospital of Zhejiang Province, Wenzhou Medical University, \\ Linhai 318000, China. Email: chenbf@enzemed.com; Jianfei Shen. Department of Cardiothoracic Surgery, Taizhou Hospital of Zhejiang Province, \\ Wenzhou Medical University, Linhai 318000, China. Email: jianfei051@163.com.
}

\begin{abstract}
Situs inversus totalis (SIT) is a rare congenital disease, with an adult incidence rate ranging from approximately 1:5,000 to 1:10,000. SIT with lung cancer is rarely reported. Most surgeons have no clinical experience with SIT. We reported the experience of a patient with SIT combined with right upper lung cancer, who underwent video-assisted thoracoscopic surgery (VATS), and provide useful information for the reader to deal with this rare situation.
\end{abstract}

Keywords: Situs inversus totalis (SIT); lung cancer; video-assisted thoracoscopic surgery (VATS)

Submitted Jul 30, 2017. Accepted for publication Sep 27, 2017.

doi: $10.21037 /$ jtd.2017.10.61

View this article at: http://dx.doi.org/10.21037/jtd.2017.10.61

\section{Introduction}

Situs inversus totalis (SIT) is a rare congenital disease, with an adult incidence rate ranging from approximately 1:5,000 to $1: 10,000$. Because of its organ position and the opposite, left and right translocation, it is also known as the mirror image (1). SIT with lung cancer is rarely reported (2). Most surgeons have no clinical experience with SIT. In addition, the thoracoscopic right upper lobe resection in these patients was not reported. Because of anatomical abnormalities, minimally invasive surgery for lung cancer resection is challenging. We reported the experience of a patient with SIT combined with right upper lung cancer, who underwent video-assisted thoracoscopic surgery (VATS), and provide useful information for the reader to deal with this rare situation.

\section{Case presentation}

A 47-year-old man was found to have type 1 situs viscerum inverses (Figure 1) with a $2.5 \mathrm{~cm}$ ground-glass opacity (GGO) of the right upper lobe via computed tomography (CT) in a healthy checkup (Figure 2A). SIT was observed with a right-sided position of the heart and aortic arch (Figure 2B); in addition, it was observed on the spleen and stomach on the right side, and the liver on the left (Figure 2C). No regional lymph nodes or distant metastasis was confirmed by imaging examination. Immediate family members of the patient have no history of SIT. The patient has no previous history of chronic sinusitis, bronchiectasis. Preoperative forced vital capacity (FVC) and forced expiratory volume in 1 second $\left(\mathrm{FEV}_{1}\right)$ were 4,450 and 3,530 $\mathrm{mL}$, respectively. The patient was chosen for a right upper lobectomy and lymph node dissection by VATS.

\section{Operational techniques}

The patient received general anesthesia with dual-lumen endotracheal intubation (2) and was placed in the left lateral decubitus position. A three-port VATS technique was used (3); the major operation hole was a 3-cm mini-incision in the 4th intercostal space of the anterior axillary line, a $1-\mathrm{cm}$ incision was made as the thoracoscopic observation hole in the 7th intercostal space of the midaxillary line, and a 1-cm incision was made as an assisted operation hole in the 7 th intercostal space of the scapular line. The rightsided position of the heart and aortic arch, and the two lobes in the right lung, were observed in operation (Figure 3). A $2.5-\mathrm{cm}$ nodule was found in the right upper lobe with 
local pleural indentation (Figure 3C), which underwent wedge resection. Frozen section examination provided "lung invasive adenocarcinoma" and then right upper lobectomy and mediastinal lymphadenectomy were performed. VATS lobectomy: right upper lobe (Figure 4).

First, the interlobular fissure was divided and the pulmonary artery exposed. The next structures to be divided was the anterior segmental artery and apical artery. Then, the posterior subsegmental artery was divided. Following the division of the superior pulmonary vein (SPV) [the superior and inferior pulmonary vein (IPV) form a common branch] (Figure 3A), the right upper lobe bronchus was exposed and divided.

Throughout the procedure, systematic lymph node sampling was conducted at levels 5, 7, 9, 10,11 and 12. At the end of the operation, two chest tubes $(26 \mathrm{~F})$ were placed within the hemithorax: one in the 7 th intercostal space of the midaxillary line and axillary line, and another in the 4th intercostal space of the anterior axillary line.

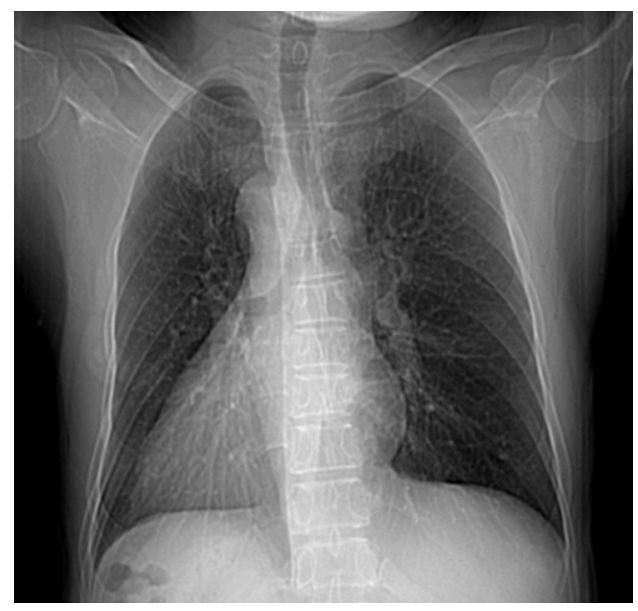

Figure 1 Chest X-ray showing dextrocardia.
The operating time was $108 \mathrm{~min}$, and the intraoperative blood loss was $98 \mathrm{~mL}$. No postoperative complications occurred. Five days later, the patient was discharged. Final pathology revealed a T2aNOM0 lung invasive adenocarcinoma.

\section{Conclusions}

VATS is the first-line strategy as therapy for early-stage, nonsmall cell lung carcinoma (NSCLC). Lobectomy for lung cancer by VATS in SIT patients has been rarely reported (5). Yoshida et al. described a right lower lobectomy using VATS in 2013, and Koichi et al. described a right upper lobectomy by thoracotomy in $2008(1,6)$; however, VATS treatment of SIT with right upper lung cancer has not been reported.

Due to anatomical abnormalities caused by SIT, double-lumen endotracheal intubation during anesthesia needs to be reversed (2). SIT patients receive unilateral lung surgery are supposed to accept left double-lumen endotracheal intubation. However, in our patient, depending on the preoperative CT, we discovered the length of the right main bronchus equivalent to the normal left main bronchus. Therefore, the anesthesiologists selected a right-sided double-lumen tube to be employed in this case. Meanwhile, the patient received the traditional left upper-lobe resection procedure.

As the SIT transposition is often combined with other deformities, before surgery, we must carefully evaluate the pulmonary artery, vein and bronchial anatomy to avoid intraoperative bleeding and transfer thoracotomy. A preoperative CT revealed that the right superior and inferior pulmonary veins form a common branch in this patient, and this was confirmed during the surgery. Application of three-dimensional (3D) CT reconstruction in these patients may help prevent hemorrhagic complications
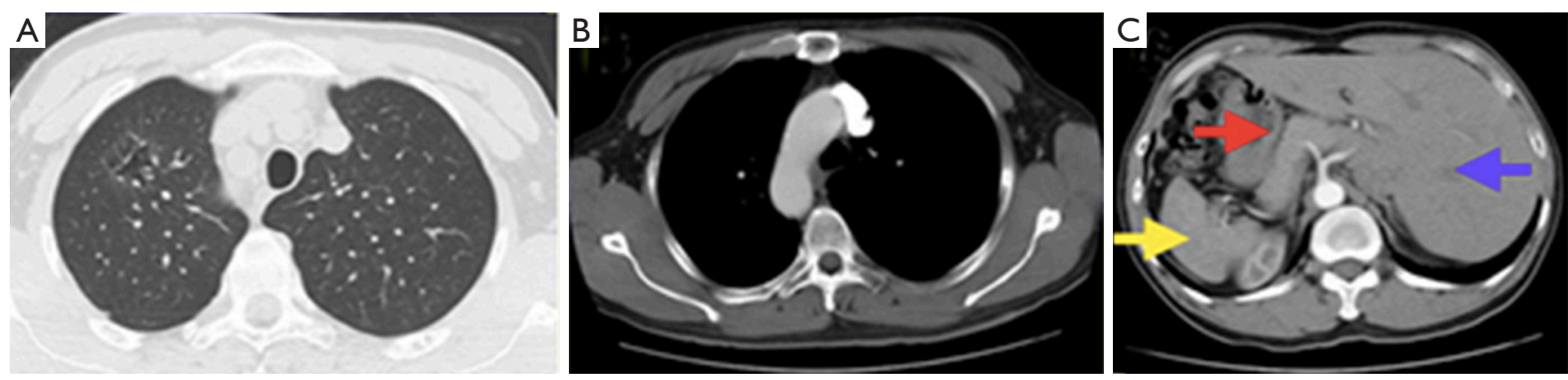

Figure 2 CT of the thorax and abdomen confirmed (A) the ground-glass opacity of the right upper lobe, (B) SIT with the aortic arch, (C) spleen (yellow arrow) and stomach (red arrow) and liver (blue arrow). CT, computed tomography; SIT, situs inversus totalis. 

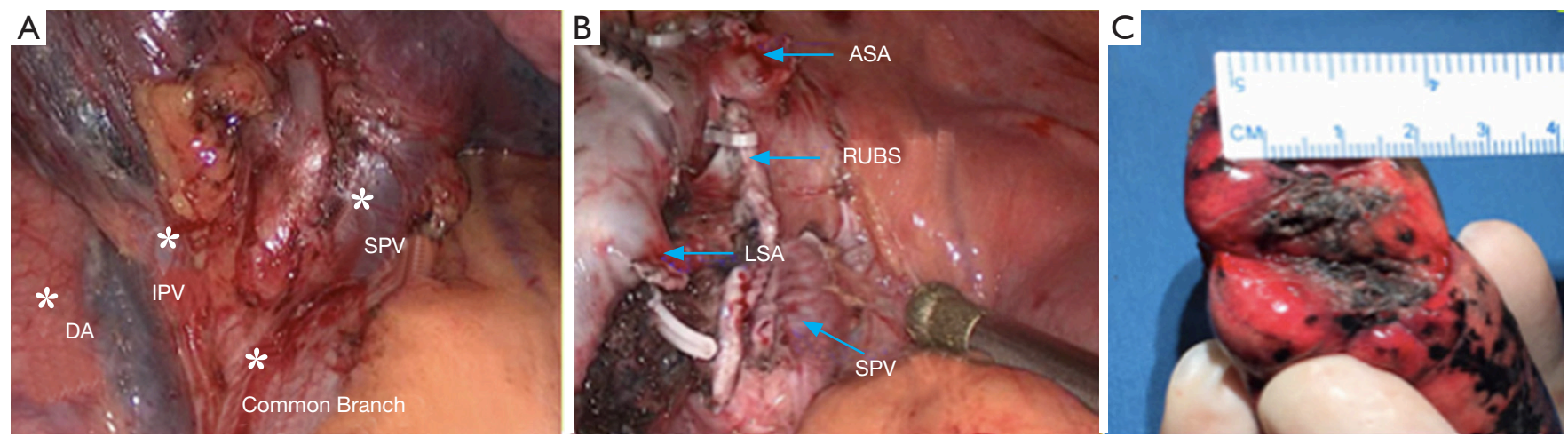

Figure 3 Intraoperative view: (A) SPV and IPV form a common branch; (B) intraoperative view after right upper lobectomy: DA; RUBS; ASA; LSA; (C) surgical specimen. *, making the sites of SPV, IPV and DA; SPV, superior pulmonary vein; IPV, inferior pulmonary vein; DA, descending aorta; RUBS, right upper bronchus stump; ASA, apical segmental artery; LSA, lingual segment artery.

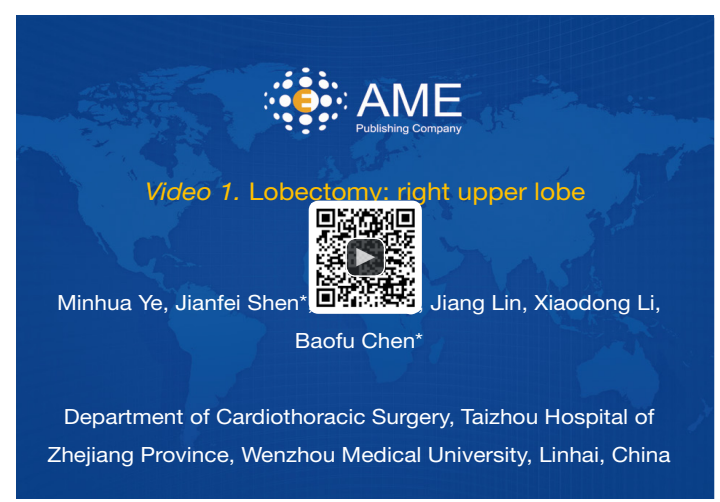

Figure 4 Lobectomy: right upper lobe (4).

Available online: http://www.asvide.com/articles/1826

for surgical treatment of lobectomy (7).

Our experience suggests that VATS treatment of SIT with lung cancer is feasible, but preoperative staff need to carefully assess whether patients have other anatomical abnormalities.

\section{Acknowledgements}

None.

\section{Footnote}

Conflicts of Interest: The authors have no conflicts of interest to declare.

Informed Consent: Written informed consent was obtained from the patient for publication of this manuscript and any accompanying images.

\section{References}

1. Yoshida M, Hino H, Machida H, et al. Video-assisted thoracic surgery lobectomy for lung cancer in a patient with complete situs inversus. Gen Thorac Cardiovasc Surg 2013;61:155-9.

2. Abdillah NJ, Wang YQ, Chen Y, et al. Lobectomy for lung cancer by video-assisted thoracic surgery in situs inversus. Asian Cardiovasc Thorac Ann 2017;25:219-21.

3. Hansen HJ, Petersen RH, Christensen M. Video-assisted thoracoscopic surgery (VATS) lobectomy using a standardized anterior approach. Surg Endosc 2011;25:1263-9.

4. Ye M, Shen J, Kong M, et al. Lobectomy: right upper lobe. Asvide 2017;4:507. Available online: http://www.asvide.com/ articles $/ 1826$

5. Grapatsas K, Piyis A, Neofotistos K, et al. A patient with situs inversus totalis and lung cancer-a rare combination. Ann Transl Med 2016;4:450.

6. Koichi F, Hiroyuki S, Hisashi H, et al. Lung resection for pulmonary carcinoma in a patient with complete situs inversus. Available online: https://www.jstage.jst.go.jp/ article/jacsurg/22/5/22_5_850/_article

7. Safdie FM, Sanchez MV, Sarkaria IS. Prevention and management of intraoperative crisis in VATS and open chest surgery: how to avoid emergency conversion. J Vis Surg 2017;3:87.

Cite this article as: Ye M, Shen J, Kong M, Lin J, Li X, Chen B. Video-assisted thoracoscopic surgery right upper lobectomy in a situs inversus totalis patient. J Thorac Dis 2017;9(11):E1018E1020. doi: 10.21037/jtd.2017.10.61 